\title{
Structure and Physiological Activity
}

\section{By John Pryde, Lecturer in Physiological Chemistry, University College, Cardiff}

\section{A} SURVEY of the road which the biochemist has trodden during the past twenty-five years reveals a plenitude of milestones whereby the rate and extent of his forward march may be judged. The year 1910 was within a short span of the birth of his science ; only four years earlier, the Biochemical Journal had first seen the light of day. The records of this now twenty-nine-yearold journal bear witness to the immense expansion and deepening, during the past quarter of a century, of our knowledge of the laboratories of the living cell, and there are many similar records to be found in other countries. New light has come from all sides on the chemical processes of the organism in health and disease, in life and in death. Innumerable new substances and previously unknown phenomena have been discovered and added to the ever-increasing physicochemical complexities of living cells and tissues.

Yet despite this immense accumulation of detail, the answers to these persistent questions"Just what happens in this particular living cell, and how does it happen ?"-- -seem to elude us. Consider for a moment the contraction of a group of muscle cells, surely one of the most universal processes of animal activity. In 1907 appeared Fletcher and Hopkins's 62-page paper on "Lactic Acid in Amphibian Muscle", the first really effective attack on the problem of the possible role of this acid in the contractile process. At that time, lactic acid was the only known 'intermediate' substance supposed to play a part in the phenomenon. Now turn to the list of substances, many then unknown, revealed to-day as participating in this apparently simple process-hexose diphosphoric and monophosphoric acids, $\alpha$-glycerophosphoric acid, phosphoglyceric acid, adenylic acid, phosphoric and pyrophosphoric acids, phosphagen or creatine phosphoric acid, pyruvic acid, methyl glyoxal, glyceraldehyde, dihydroxyacetone, and . . . but who of us is bold enough to write finis to this catalogue? In the numerous attempts to build up the chemical jig-saw here provided, many new and interesting and doubtless important facts have obviously been revealed, but we still seem to be almost as far as we were in 1907 from forming a really comprehensive picture of the sequence of events in a contracting muscle. Indeed one investigator has suggested that all the chemical transformations so far studied are really recovery processes, and do not directly participate in the contractile phase.
The present position of muscle chemistry will serve to illustrate one type of problem which the biochemist is studying in his attempt to provide an answer to the questions already mentioned. There are many similar problems. Most of them afford as much encouragement as the muscle problem to those who aspire to conquer fresh fields! There are also, however, problems of a rather different type which likewise concern the biochemist. A scrutiny of the records of the investigations here referred to conveys a greater sense of immediate achievement than that which is derived from a consideration of the muscle and similar problems. Here we have revealed to us the nature, and in many cases the detailed structure, of compounds of immense physiological potency, compounds some of which determine the issue between life and death, whilst others possess well-nigh incredible potentialities for the wellbeing, in some cases the ill-being, of living organisms. All seem to exercise their functions in the minutest traces, a fact which has added greatly to the difficulties of their isolation and identification, but during the last decade the progress achieved in this direction is highly impressive.

Most of the compounds now to be discussed may be assigned to one of two classes, hormones or vitamins-hormones if they are elaborated within the animal by specific tissues which derive their 'raw material' from the food supply, vitamins if they must be supplied pre-formed in the food. stuffs. This classification cannot be applied too rigidly, but it will serve. As we shall see, certain vitamins can be formed by the animal body, from precursors of very closely related chemical structure but devoid of the characteristic physiological properties of the vitamin. In the absence of the vitamin, such precursors assume the rôle of indispensable food constituents. To the chemist and the biochemist, the smallness of the amount of these substances which the animal requires is a matter of the greatest interest, but these investigators derive their main sense of achievement in this field from the ability to put down on paper the constitution or structure of the substances concerned. How the substances produce their specific effects is a secret so far guarded by Nature even more efficiently than that of the muscle Nonetheless, science is for the present well content with having established the existence and structure of at least some of these enormously potent 
substances upon which animal life depends for its very existence.

The constitutions of the known hormones or vitamins do not afford, in the absence of empirical information from other sources, a priori grounds for deducing their physiological activities, or for differentiating between an active compound and some closely related inactive compound with a structure but slightly different from that of the active one. Such a priori deductions would necessitate much more fundamental correlations between structure and physiological activity than are yet available. But, in actual fact, empirical information is rapidly accumulating, and as it becomes co-ordinated it is found possible to make certain prognostications regarding structure and physiological activity, to suggest, for example, what type of structure is likely to possess a particular activity, and possibly how such activity may be modified when certain substituent groups are added to or taken away from the basic structure.

These general considerations are well illustrated by a series of compounds, including both hormones and vitamins, which have in common as a structural basis the condensed benzene ring system which the organic chemist calls phenanthrene. The reduced phenanthrene system plus a fourth five-membered carbon ring forms the cholane nucleus; this nucleus is found in the bile acids and in cholesterol and the other sterols. One of these sterols, ergosterol, $\mathrm{C}_{28} \mathrm{H}_{44} \mathrm{O}$, when subjected to the action of ultra-violet radiation, undergoes a sequence of transformations, and amongst the products formed under suitable conditions is calciferol, which is isomeric with the original ergosterol. Calciferol, which has been isolated in a pure crystalline condition, is vitamin $\mathrm{D}$, the anti-rachitic vitamin essential for adequate ossification and for the control of calcium metabolism in the animal organism. The structure of ergosterol is not yet ascertained in all its details, but existing evidence is in accord with the formula shown in skeleton below.

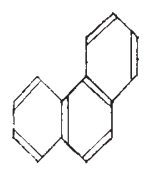

Phenanthrene

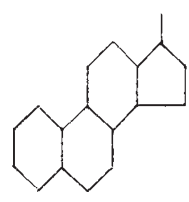

Cholane nucleus

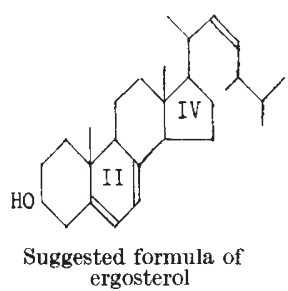

ergosterol
It would appear that the transformation of ergosterol into calciferol involves the shift of the double bonds in ring II in the direction of the double bond in the side-chain of ring IV. But a final decision cannot yet be reached. The vitamin calciferol may be ingested as such by the animal. On the other hand, it may be formed within the animal from previously ingested ergosterol. This synthesis in vivo of the vitamin from its nearly related precursor has been proved experimentally to occur when ultra-violet radiation impinges on the skin. The active rays have sufficient penetrative power to effect the transformation. We have here the basis of the curative action in rickets of ultra-violet rays of solar or other origin.

A structure of the cholane type is found in a series of recently discovered hormones possessing remarkably interesting properties. These are the sex hormones which determine the secondary sex characters of animals. Three have been definitely characterised, the female follicular hormone or œstrone, the female corpus luteum hormone or luteosterone, and the male testicular hormone or androsterone. All three show close structural relationships with each other and with the bile acids and cholesterol, amongst which their biological precursors are likely to be found. The accepted structures of the three hormones are given below.

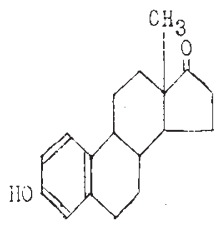

Fstrone

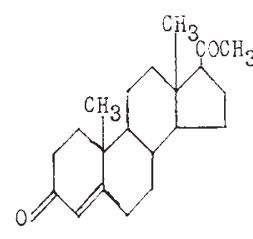

Luteostcrone

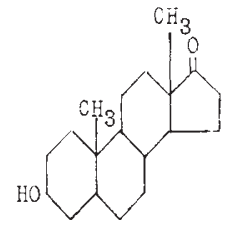

Androsterone
A development of exceptional interest in relation to structure and physiological activity is the preparation by synthetic means of artificial œestrogenic substances, that is, of substances not found naturally, but which possess an activity similar to that of cestrone. One of these is 1-keto-1 $: 2: 3: 4$-tetrahydrophenanthrene, another is a derivative of the hydrocarbon $1: 2: 5: 6$ dibenzanthracene, of which further mention will be made.
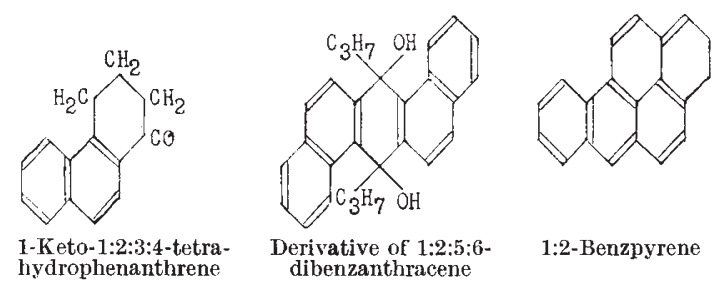

The phenanthrene structure, common to all the substances so far considered, will be seen in the formulæ shown above. These two synthetic œstrogenic substances, it is of interest to note, do not show any androsterone activity.

In the hydrocarbon $1: 2: 5: 6$-dibenzanthracene, we encounter one of the very interesting and, 
from human standards, very sinister synthetic carcinogenic hydrocarbons. This hydrocarbon produces, when applied to the skin of mice and rats, tumours which can be transplanted and are in all respects similar to malignant cancerous growths. Yet the addition of two $n$-propyl and two hydroxyl groups converts it to an œestrogenic substance! Dibenzanthracene is but one of a number of synthetic carcinogenic hydrocarbons. All of these contain the phenanthrene nucleus. Moreover, the long-recognised cancer-producing action of certain tars has now been traced to the presence of a hydrocarbon allied to dibenzanthracene and known as $1: 2$-benzpyrene. It has been synthesised independently of its isolation from coal tar, and the powerful carcinogenic action of the pure substance has been amply confirmed. It is, in fact, the most potent carcinogenic substance so far discovered. It too will be seen to contain the phenanthrene nucleus.

Suggestive and interesting as is the rôle of the phenanthrene nucleus already outlined, three further references are required to complete the tale. First, a series of toxic cardiac-stimulating glucosides is known These are derived from various plants including those of the species

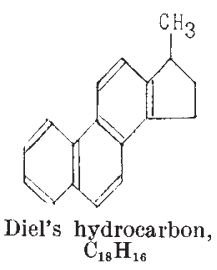
Digitalis (foxglove) and Strophanthus. The aglucones (that is, the non-sugar parts) of digitoxin, strophanthin and several other closely related sub. stances embody structures of four carbon rings which ally them closely to the sterols. For example, the dehydrogenation of the aglucone strophanthidin with selenium yields the well-known Diel's hydrocarbon, $\mathrm{C}_{18} \mathrm{H}_{16}$, of which the constitution has been established by synthesis. It has been prepared from several members of the cholane series, and its relationship to this series is beyond doubt. Many other structural details are known concerning the aglucones of the cardiac-stimulating glucosides and in some cases a complete structural formula can be suggested even now. Secondly, mention must be made of the toad poisons, amongst which is the Chinese drug Ch'an Su. These contain structures closely allied to those of the cardiac aglucones, and possess a characteristic action on the heart similar to that of Digitalis preparations. Lastly, brief reference may be made to the significant fact that the phenanthrene nucleus is present in some of the most powerful alkaloids, in morphine and codeine of the opium group, in the corydalis alkaloids and in colchicine (meadow saffron).

Let us leave now these inimical carcinogenic hydrocarbons, toxic glucosides, toad poisons and alkaloids and return to the kindlier theme of vitamins and hormones. From the remainder of our story there stand out two achievements. In both cases the proofs of the nature of the substances concerned-a vitamin in one case, a hormone in the other-have been clinched by syntheses. Ascorbic acid, as the anti-scorbutic vitamin $\mathrm{C}$ is now termed, and thyroxine, the iodine-containing amino acid which confers upon the secretory protein of the thyroid gland its specific function as a regulator of the metabolic rate, have both been isolated in a pure state, and both have been synthesised. As a justification for treating these two diverse substances together in this way, we may cite the exceptional brilliance of the work on the synthetic side. The structures are shown below. It will be seen that ascorbic

$$
\begin{gathered}
\mathrm{CH}_{2}(\mathrm{OH}) \cdot \mathrm{CH}(\mathrm{OH}) \cdot \mathrm{CH}-\mathrm{C}=\underset{\mathrm{C}}{\mathrm{O}-\mathrm{CO}} \\
\text { Ascorbic acid }
\end{gathered}
$$

acid is a near relative of the carbohydrates-the starting material used in its laboratory synthesis

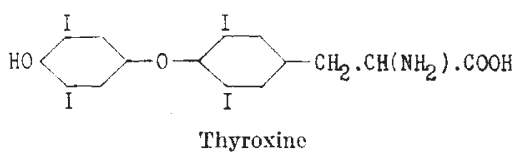

was a hexose, $d$-galactose, from which $l$-lyxose and $l$-lyxosone were obtained. Thyroxine is related to the well-known amino acid tyrosine, and the latter is almost certainly the 'raw material' used in the animal body for its formation.

The case of vitamin A, the growth-promoting vitamin, is especially interesting, since here we have another case of a vitamin which may be taken into the animal body as such, or in the form of a closely related precursor. The precursor is the remarkable, intensely coloured polyene hydrocarbon carotene, with no less than forty carbon atoms. At least four isomeric carotenes occur in natural plant sources, and all appear to give rise to vitamin $A$ in the animal body. Approximately one half of the carotene molecule goes to form vitamin $\mathrm{A}$, which almost certainly has the formula $\mathrm{C}_{20} \mathrm{H}_{30} \mathrm{O}$. The structure of the vitamin cannot yet be regarded as settled, but there is much evidence in favour of that given below.

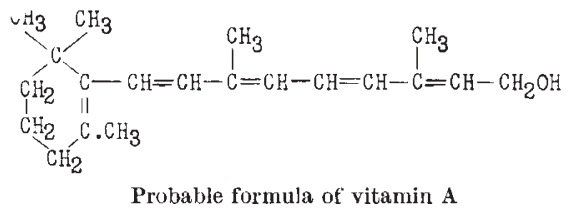

We may end our survey with a reference to advances which are being made in the elucidation 
of the structures of vitamins $B_{1}$ and $B_{2}$. Vitamin $\mathrm{B}_{1}$ has the formula $\mathrm{C}_{12} \mathrm{H}_{18} \mathrm{O}_{2} \mathrm{~N}_{4} \mathrm{~S}$. There appear to be two ring systems present; one a glyoxaline or pyrimidine, the other a pyrrole containing a substituent sulphur. Interest in vitamin $\mathrm{B}_{2}$ has been greatly stimulated by the recent discovery that the flavins, which are yellow water-soluble dyes present in both animal and vegetable sources, possess intense vitamin $B_{2}$ activity. The flavins are derivatives of alloxazine, and a substance stated to be identical with lactoflavin of milk whey has already been synthesised. It possesses the complex ring systems shown. So discovery<smiles></smiles>

One of ring systems of
vitamin $B$

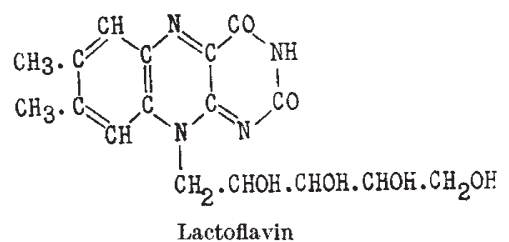

Lactoflavin marches onwards! Who shall say what additional structural formulæ will be recorded in a similar survey after the lapse of another quarter of a century?

\section{Diet and Disease}

By Prof. Stuart J. Cowerr, Professor of Dietetics, St. Thomas's Hospital Medical School, London

$\mathrm{T}$ HE twenty-five years of His Majesty's reign which are now being celebrated correspond remarkably closely with the establishment of a new era in the science of nutrition. At the opening of the twentieth century, attention was being focused on the quantitative relations of the energy exchanges of the body and on the metabolism within the body of the proteins, fats and carbohydrates of the food. The physiologists and chemists working at these problems were making most valuable contributions to the body of knowledge concerning the processes of nutrition, but such contributions were for the most part not of such a nature as to afford obvious clues either to the origin of or to the treatment of disease. The second decade of the twentieth century witnessed the rapid development of the view that the adequate nutrition of an animal depended on the presence in its food of hitherto unsuspected elements. The absence of such essential elements from a diet was proved to result regularly in the appearance of predictable signs of disease, and the fundamentally new idea of deficiency diseases became gradually established in current medical teaching.

Before mentioning any of the effects which this new conception of nutrition has had on the problems of the prevention and treatment of disease, it will be useful to hark back to the 'pre-Georgian' era to review the current teaching of the medical profession regarding the relation of diet to disease. The fact that faulty diets are often the direct or indirect cause of disease was of course fully recognised, as it had been for many centuries. But there was little precise knowledge available to enable definite diseases to be ascribed to specific dietetic errors. Over-eating was regarded as predisposing to many gastro-intestinal diseases, gout and raised blood pressure, and under-eating was considered to render the body more liable to invasion by harmful bacteria. The idea of lack of balance between the various classes of foodstuffs, for example, relative excess or deficiency of protein, carbohydrate or fat was looked upon as at least an important contributory cause of disease. In the case of scurvy, it was already taught that the absence from the diet of some principle which was present in fresh foods but not in stale foods contributed largely to the production of the disorder. Otherwise the production of disease by faulty diet was largely related to the presence of toxins, pathogenic bacteria or living parasites in food which had become accidentally contaminated.

With regard to the practical dietetic management of diseases now known to be due to specific dietetic faults, the degree of divergence between the methods of twenty-five years ago and of the present day is distinctly less than would have been expected from a consideration of the knowledge available then and now. Specific remedies in medicine have again and again been discovered empirically, and this is true in the realm of dietetics. The treatment recommended twentyfive years ago by at least some enlightened authorities for many of the diseases now spoken of as deficiency diseases would prove satisfactory enough to-day, although such treatment was based on no actual knowledge of the dietetic factors involved. Scurvy was treated by giving fresh fruit and fresh vegetables, rickets and osteomalacia by giving cod liver oil and milk, beriberi by increasing the 'nitrogenous' constituents and diminishing the carbohydrate of the diet-some individuals were even claiming that it could be prevented by adding rice polishings to the diet of highly polished rice which was usually eaten in districts where this disease occurred-and finally pellagra was 